\title{
Remark about non-relativistic p-brane
}

\author{
Josef Klusoñ ${ }^{\mathrm{a}}$ \\ Department of Theoretical Physics and Astrophysics, Faculty of Science, Masaryk University, Kotlářská 2, 61137 Brno, Czech Republic
}

Received: 11 September 2017 / Accepted: 21 December 2017 / Published online: 13 January 2018

(C) The Author(s) 2018. This article is an open access publication

\begin{abstract}
We define a different non-relativistic limit of pbrane with the help of canonical form of the p-brane action. We discuss the properties of these actions and their symmetries.
\end{abstract}

\section{Introduction}

Recently, a new interesting formulation of non-relativistic theories was proposed in [1] and further elaborated in [2-5]. ${ }^{1}$ These theories belong to the class of systems with reduced symmetries that were analyzed recently from different point of views. A very important subject is non-relativistic holography, which is a very useful tool for the study of strongly correlated systems in condensed matter; for a recent review see [6]. Non-relativistic symmetries also have a fundamental meaning in the recent proposal of renormalizable quantum theory of gravity known today as Hořava-Lifshitz gravity [7]; for a recent review and an extensive list of references, see [8]. There is also an interesting connection between HořavaLifshitz gravity and Newton-Cartan gravity $[9,10]$. In fact, Newton-Cartan gravity and its relation to different limits was also studied recently in a series of papers [11-17].

Another possibility to define non-relativistic theories is to perform a non-relativistic limit on the level of the action for a particle, string or p-brane. The first example of such an object was the non-relativistic string introduced in $[18,19]$. These actions were obtained by a non-relativistic "stringy" limit, where the time direction and one spatial direction along the string are large. The stringy limit of the superstring in $A d S_{5} \times S^{5}$ was also formulated in [20] and it was argued here that it provides another soluble sector of the AdS/CFT correspondence; for related work, see $[25,26]$. The non-relativistic

\footnotetext{
${ }^{1}$ For a recent very nice proposal of non-relativistic string, see lso [29].

a e-mail: klu@physics.muni.cz
}

limit was further extended to the case of higher dimensional objects in string theory, as for example p-branes [21-24].

It is important to stress that there is also a non-relativistic limit of the relativistic string where only the time direction is large. In this case the non-relativistic string does not vibrate and it represents a collection of non-relativistic massless particles.

All these limits were very carefully analyzed in [1] where the general procedure to implement the non-relativistic limit for a different relativistic action was proposed. The main idea is to start with an action for a relativistic extended object with coordinates $X$,

$S=\int \mathcal{L}(X)$

and assume that the Lagrangian density is pseudo-invariant under the set of relativistic symmetries $\delta_{R}$,

$\delta_{R} \mathcal{L}=\partial_{\mu} F^{\mu}$.

Then in order to find a non-relativistic limit of this action we introduce the dimensionless parameter $\omega$ and we define different non-relativistic limits by appropriately rescaling the coordinates and parameters in the Lagrangian density. Then we can presume that the Lagrangian density and the symmetry transformation can be expanded in powers of $\omega$,

$$
\begin{aligned}
\delta_{R} & =\delta_{0}+\omega^{-2} \delta_{-2}+\cdots, \\
\delta \mathcal{L} & =\omega^{2} \mathcal{L}_{2}+\mathcal{L}_{0}+\omega^{-2} \mathcal{L}_{-2}+\cdots, \\
F^{\mu} & =\omega^{2} F_{2}^{\mu}+F_{0}^{\mu}+\omega^{-2} F_{-2}^{\mu}+\cdots,
\end{aligned}
$$

where the first term in the expansion of the relativistic symmetry $\delta_{R}$ is the non-relativistic transformation $\delta_{0}$. Then Eq. (2) implies an infinite set of equations when we compare the expressions of the same orders in $\omega$,

$$
\begin{aligned}
& \delta_{0} \mathcal{L}_{2}=\partial_{\mu} F_{2}^{\mu}, \\
& \delta_{0} \mathcal{L}_{0}+\delta_{-2} \mathcal{L}_{2}=\partial_{\mu} F_{0}^{\mu}, \\
& \delta_{0} \mathcal{L}_{-2}+\delta_{-2} \mathcal{L}_{0}+\delta_{-4} \mathcal{L}_{2}=\partial_{\mu} F_{-2}^{\mu}
\end{aligned}
$$


The special case occurs when the Lagrangian density is invariant under the relativistic symmetry so that $F^{\mu}=0$. Then from the previous equations we see that $\mathcal{L}_{2}$ is invariant under a non-relativistic symmetry, while $\mathcal{L}_{0}$ is generally not invariant under a non-relativistic symmetry. It is further important to stress that $\mathcal{L}_{2}$ contributes to the action with the factor $\omega^{2}$ and hence gives a dominant contribution in the limit $\omega \rightarrow \infty$, while $\mathcal{L}_{0}$ remains finite and terms proportional to $\mathcal{L}_{-2}, \mathcal{L}_{-4}, \ldots$ vanish.

Since this general procedure is very interesting we think that it is useful to explore it in more detail. In particular, we would like to formulate this procedure using the canonical form of the action when we express the Lagrangian density using the corresponding Hamiltonian. It turns out that it is very useful, since it allows us to straightforwardly identify physical degrees of freedom in the limit $\omega \rightarrow \infty$. More precisely, we introduce a scaling of the non-relativistic directions at the level of the action and then we find the corresponding Hamiltonian for finite $\omega$. The corresponding canonical action is invariant under relativistic transformations by definition and we also determine the form of these transformations for rescaled variables for finite $\omega$. Then we discuss the properties of the resulting Lagrangian density in dependence on the scaling of the tension of the original p-brane and on the number of non-relativistic dimensions. We argue that the non-relativistic Lagrangian $\mathcal{L}_{0}$ is invariant under non-relativistic symmetries under the condition that the Lagrangian $\mathcal{L}_{2}$ vanish, in agreement with the general discussion in [1]. We also argue that in the case that $\mathcal{L}_{2}$ is non-zero the variation of the Lagrangian density $\mathcal{L}_{0}$ under non-relativistic transformations exactly cancels the variation $\delta_{-2} \mathcal{L}_{2}$. On the other hand in this case we are not quite sure how to deal with the divergent term in the Lagrangian which, however, can be canceled when we allow that $\mathrm{p}$-brane couples to an appropriate $p+1$-form field exactly as in [24]. However, the fact that there is a background $p+1$ form breaks the original relativistic symmetry to the subgroup that leaves this background field invariant and hence the symmetry group is reduced. More precisely, in the case that we cancel the divergent term the Lagrangian density $\mathcal{L}_{2}$ is zero and hence the Lagrangian density $\mathcal{L}_{0}$ has to be invariant under a reduced group of symmetries. Of course, there is an exception, which is a fundamental string when it can be shown that in the flat space-time the Lagrangian density $\mathcal{L}_{2}$ is a total derivative and hence can be ignored [1]. We also determine a Hamiltonian constraint for the non-relativistic p-brane and we show that it is linear in the momenta.

As the final part of our work we focus on a particle-like limit of the p-brane when only the time direction is large. Using the canonical form of the action we easily find the corresponding Lagrangian and we show that it is invariant under Galilean transformations.
This paper is organized as follows. In Sect. 2 we introduce a non-relativistic limit of the canonical form of the action and discuss symmetries of the theory. In Sect. 3 we analyse the particular case when the matrix $\tilde{G}_{i j}$, whose explicit form is defined in (8), is non-singular. In Sect. 4 we discuss the possibility to eliminate the divergent term by a coupling of the $\mathrm{p}$-brane to a $p+1$ form and we derive the corresponding non-relativistic Hamiltonian. In Sect. 5 we take a particlelike non-relativistic limit of the p-brane and the fundamental string. Finally, in conclusion (6) we outline our result and suggest a possible extension of this work.

\section{Non-relativistic limit of p-brane canonical action}

In this section we formulate our proposal to define a nonrelativistic p-brane using the canonical form of the action. The starting point is an action for a relativistic p-brane,

$$
S=-\tilde{\tau}_{p} \int d^{p+1} \xi \sqrt{-\operatorname{det} \mathbf{A}_{\alpha \beta}},
$$

$\mathbf{A}_{\alpha \beta}=\eta_{A B} \partial_{\alpha} \tilde{x}^{A} \partial_{\beta} \tilde{x}^{B}$,

where $\tilde{x}^{A}, A=0, \ldots, d$ labels the embedding of the p-brane in the target space-time and where $\eta_{A B}=\operatorname{diag}(-1, \underbrace{1 \ldots, 1})$.

It is important to stress that the action is invariant under a relativistic symmetry,

$\tilde{x}^{\prime A}=\tilde{\Lambda}_{B}^{A} \tilde{x}^{B}+b^{A}, \quad \tilde{\Lambda}_{A}^{C} \eta_{C D} \tilde{\Lambda}_{B}^{D}=\eta_{A B}$,

where $\tilde{\Lambda}_{B}^{A}, b^{A}$ are constants. The action (5) was the starting point for the definition of the non-relativistic limit that was presented in [1]. As was argued there it is possible to define $p+1$ different non-relativistic limits according to the number of embedding coordinates $(0, \ldots, p+1)$, which are rescaled. Explicitly, we have

$$
\begin{aligned}
\tilde{x}^{\mu} & =\omega X^{\mu}, \quad \mu=0, \ldots, q, \quad \tilde{x}^{M}=X^{M}, \\
M & =q+1, \ldots, d, \quad \tilde{\tau}_{p}=\frac{\tau_{p}}{\omega^{k_{q}}},
\end{aligned}
$$

where the number $k_{q}$ depends on the form of the nonrelativistic limit. Inserting (7) into the definition of the matrix $\mathbf{A}_{\alpha \beta}$ we see that it has the form

$$
\begin{aligned}
& \mathbf{A}_{\alpha \beta}=\omega^{2} \tilde{G}_{\alpha \beta}+\mathbf{a}_{\alpha \beta}, \\
& \tilde{G}_{\alpha \beta} \equiv \partial_{\alpha} X^{\mu} \partial_{\beta} X_{\mu}, \quad \mathbf{a}_{\alpha \beta}=\partial_{\alpha} X^{M} \partial_{\beta} X_{M} .
\end{aligned}
$$

Observe that we can write the matrix $\mathbf{A}_{\alpha \beta}$ as

$$
\begin{aligned}
\mathbf{A}_{\alpha \beta}= & G_{A B} \partial_{\alpha} X^{A} \partial_{\beta} X^{B}=G_{\mu \nu} \partial_{\alpha} X^{\mu} \partial_{\beta} X^{\nu} \\
& +G_{M N} \partial_{\alpha} X^{M} \partial_{\beta} X^{N},
\end{aligned}
$$

where $G_{\mu \nu}=\omega^{2} \eta_{\mu \nu}, \quad G_{M N}=\delta_{M N}$.

Our proposal is to define a non-relativistic limit with the help of the canonical form of the action. To do this we find the 
Hamiltonian formalism for the p-brane for finite $\omega$ and take the limit $\omega \rightarrow \infty$ after we derive canonical form of the action. Note that $k_{q}$ is an integer number that will be determined by requirement that there are terms in the Lagrangian density at most quadratic at $\omega^{2}$. Using (8) we find the following conjugate momenta:

$$
\begin{aligned}
& p_{\mu}=-\frac{\tau_{p}}{\omega^{k_{q}}} \omega^{2} \partial_{\beta} X_{\mu}\left(\mathbf{A}^{-1}\right)^{\beta 0} \sqrt{-\operatorname{det} \mathbf{A}}, \\
& p_{M}=-\frac{\tau_{p}}{\omega^{k_{q}}} \partial_{\alpha} X_{M}\left(\mathbf{A}^{-1}\right)^{\alpha 0} \sqrt{-\operatorname{det} \mathbf{A}} .
\end{aligned}
$$

Then it is easy to see that the bare Hamiltonian is equal to zero,

$H_{B}=\int d^{p} \xi\left(p_{\mu} \partial_{0} X^{\mu}+p_{M} \partial_{0} X^{M}-\mathcal{L}\right)=0$,

while we have the following collection of the primary constraints:

$$
\begin{aligned}
\mathcal{H}_{i} & =p_{\mu} \partial_{i} X^{\mu}+p_{M} \partial_{i} X^{M} \approx 0, \\
\tilde{\mathcal{H}}_{\tau} & =\frac{1}{\omega^{2}} p_{\mu} \eta^{\mu \nu} p_{v}+p_{M} p^{M}+\frac{\tau_{p}^{2}}{\omega^{2 k_{q}}} \operatorname{det} \mathbf{A}_{i j} \approx 0,
\end{aligned}
$$

so that the Lagrangian density has the form

$$
\begin{aligned}
\mathcal{L}= & p_{\mu} \partial_{0} X^{\mu}+p_{M} \partial_{0} X^{M} \\
& -\lambda^{\tau}\left(\frac{1}{\omega^{2}} p_{\mu} \eta^{\mu \nu} p_{\nu}+p_{M} p^{M}+\frac{\tau_{p}^{2}}{\omega^{2 k_{q}}} \operatorname{det} \mathbf{A}_{i j}\right) \\
& -\lambda^{i}\left(p_{\mu} \partial_{i} X^{\mu}+p_{M} \partial_{i} X^{M}\right) .
\end{aligned}
$$

Note that $i=1, \ldots, q$ labels the spatial world-volume directions of the p-brane. Let us now discuss the Lorentz transformations (6) in more detail. It is instructive to write them in the form

$$
\left(\begin{array}{c}
\tilde{x}^{\prime \mu} \\
\tilde{x}^{\prime M}
\end{array}\right)=\left(\begin{array}{cc}
\tilde{\Lambda}_{\rho}^{\mu} & \tilde{\Lambda}_{K}^{\mu} \\
\tilde{\Lambda}_{\rho}^{M} & \tilde{\Lambda}_{K}^{M}
\end{array}\right)\left(\begin{array}{c}
\tilde{x}^{\rho} \\
\tilde{x}^{K}
\end{array}\right) .
$$

If we replace the original variables with the rescaled ones we obtain

$$
\begin{aligned}
X^{\prime \mu} & =\tilde{\Lambda}_{\nu}^{\mu} X^{\nu}+\frac{1}{\omega} \tilde{\Lambda}^{\mu}{ }_{M} X^{M}, \\
X^{\prime M} & =\omega \tilde{\Lambda}_{\nu}^{M} X^{v}+\tilde{\Lambda}_{N}^{M} X^{N},
\end{aligned}
$$

where $\tilde{\Lambda}_{B}^{A}$ has to obey the equation

$$
\tilde{\Lambda}_{C}^{A} \tilde{\Lambda}_{D}^{B} \eta_{A B}=\eta_{C D}
$$

It is natural to require that the transformation rule for $X^{M}$ is finite in the limit $\omega \rightarrow \infty$ and hence we perform the following rescaling:

$\tilde{\Lambda}_{v}^{M}=\frac{1}{\omega} \Lambda_{v}^{M}$

Further, from (15) we see that $\tilde{\Lambda}_{v}^{\mu}, \tilde{\Lambda}_{N}^{M}$ are not rescaled:

$\tilde{\Lambda}_{v}^{\mu}=\Lambda_{v}^{\mu}, \quad \tilde{\Lambda}_{N}^{M}=\Lambda_{N}^{M}$.
On the other hand if we decompose (16) into corresponding components we obtain

$$
\begin{gathered}
\Lambda_{\mu}^{\rho}{ }_{\mu} \eta_{\rho \sigma} \Lambda_{v}^{\sigma}+\frac{1}{\omega^{2}} \Lambda_{\mu}^{M} \delta_{M N} \Lambda_{\nu}^{N}=\eta_{\mu \nu}, \\
\Lambda_{\rho}^{\mu} \eta_{\mu \nu} \tilde{\Lambda}_{M}^{v}+\frac{1}{\omega} \Lambda_{\rho}^{N} \delta_{N K} \Lambda_{M}^{K}=0, \\
\tilde{\Lambda}_{M}^{\mu} \eta_{\mu \nu} \Lambda_{\rho}^{v}+\frac{1}{\omega} \Lambda_{M}^{N} \delta_{N K} \lambda_{\rho}^{K}=0, \\
\frac{1}{\omega^{2}} \Lambda_{M}^{\mu}{ }_{M} \eta_{\mu \nu} \Lambda_{N}^{\nu}+\Lambda_{M}^{K} \delta_{K L} \Lambda_{N}^{L}=\delta_{M N},
\end{gathered}
$$

and we see that we have to demand the following scaling rule for $\tilde{\Lambda}_{M}^{\mu}$ :

$\tilde{\Lambda}_{N}^{\mu}=\frac{1}{\omega} \Lambda_{M}^{\mu}$

Using these results in (15) we obtain the final form of the Lorentz transformations for the rescaled variables:

$$
\begin{aligned}
X^{\prime \mu} & =\Lambda_{\nu}^{\mu} X^{\nu}+\frac{1}{\omega^{2}} \Lambda_{M}^{\mu} X^{M}, \\
X^{\prime M} & =\Lambda_{N}^{M} X^{N}+\Lambda_{\nu}^{M} X^{\nu}
\end{aligned}
$$

or in its infinitesimal form: $\Lambda_{v}^{\mu}=\delta_{v}^{\mu}+\omega_{v}^{\mu}, \Lambda_{M}^{\mu}=$ $\lambda^{\mu}{ }_{M}, \Lambda_{v}^{M}=\lambda_{v}^{M}, \Lambda_{N}^{M}=\delta_{N}^{M}+\omega_{N}^{M}$,

$$
\begin{gathered}
\delta X^{\mu}=X^{\prime \mu}-X^{\mu}=\omega^{\mu}{ }_{\nu} X^{\nu}+\frac{1}{\omega^{2}} \lambda^{\mu}{ }_{M} X^{M}, \\
\delta X^{\prime M}=X^{\prime M}-X^{M}=\omega^{M}{ }_{N} X^{N}+\lambda_{\nu}^{M} X^{v},
\end{gathered}
$$

so that

$$
\begin{aligned}
\delta_{0} X^{\mu} & =\omega^{\mu}{ }_{\nu} X^{v}, \quad \delta_{0} X^{M}=\omega_{N}^{M} X^{N}+\lambda^{M}{ }_{\nu} X^{v}, \\
\delta_{-2} X^{\mu} & =\Lambda^{\mu}{ }_{M} X^{M} .
\end{aligned}
$$

It is important to stress that the parameters $\omega_{\nu}^{\mu}, \lambda_{\mu}^{M}$ can be expanded in powers of $\omega^{-2}$ so that we obtain an infinite number of terms in the expansion of the Lorentz transformations in agreement with the general definition (4). However, for our purposes the number of these terms as given above is sufficient.

Since we consider the Lagrangian density in the canonical form we also have to find the corresponding transformation rule for conjugate momenta. For simplicity we will consider an infinitesimal form of the transformation and we demand that the combination $p_{\mu} \partial_{0} X^{\mu}+p_{M} \partial_{0} X^{M}$ is invariant,

$\delta p_{\mu} \partial_{0} X^{\mu}+p_{\mu} \partial_{0} \delta X^{\mu}+\delta p_{M} \partial_{0} X^{M}+p_{M} \partial_{0} \delta X^{M}=0$,

which in the end implies the following transformation rules:

$$
\begin{aligned}
\delta p_{\mu} & =-p_{\nu} \omega_{\mu}^{\nu}-p_{M} \lambda_{\mu}^{M}, \\
\delta p_{M} & =-p_{N} \omega_{M}^{N}-\frac{1}{\omega^{2}} p_{\mu} \lambda_{M}^{\mu} .
\end{aligned}
$$

It is clear that the Lagrangian density (13) is invariant under these transformations, since it is manifestly invariant under 
Lorentz transformations and the transformation rules given above are ordinary Lorentz transformations rewritten with the help of the rescaled variables. Another situation occurs when we consider a specific form of the non-relativistic Lagrangian density and study its properties in the limit $\omega \rightarrow \infty$. It is important to stress that the Lagrangian density (13) is exact in $\omega$ and we can perform its expansion in powers of $\omega^{2}$ exactly as in [1] even for the case when the matrix $\tilde{G}_{i j}$ is singular. For simplicity we restrict ourselves to two particular cases that allow one to find a simple result, which, however, also describes the main properties of the procedure introduced above. We start with the case when the matrix $\tilde{G}_{i j}$ is non-singular.

\section{The first case: $\tilde{G}_{i j}$ is non-singular matrix}

As the first possibility we consider the case when $\tilde{G}_{i j}$ is a non-singular matrix. Note that it is a $p \times p$ matrix in the form $\partial_{i} X^{\mu} \eta_{\mu \nu} \partial_{j} X^{\nu}$ where $\partial_{i} X^{\mu}$ is a $p \times(q+1)$ matrix where $q \leq p$. In the case that $q+1=p$ we find that $\tilde{G}_{i j}$ is a non-singular matrix and we can write

$$
\operatorname{det}\left(\omega^{2} \tilde{G}_{i j}+\mathbf{a}_{i j}\right)=\omega^{2 p} \operatorname{det} \tilde{G} \operatorname{det}\left(\delta_{i}^{j}+\frac{1}{\omega^{2}} \tilde{G}^{i k} \mathbf{a}_{k j}\right) .
$$

If we choose $k_{q}=p$ we find that the Lagrangian density has the form

$$
\begin{aligned}
\mathcal{L}= & p_{\mu} \partial_{0} X^{\mu}+p_{M} \partial_{0} X^{M} \\
& -\lambda^{\tau}\left(\frac{1}{\omega^{2}} p_{\mu} \eta^{\mu v} p_{v}+p_{M} p^{M}+\tau_{p}^{2} \operatorname{det} \tilde{G}_{i j}\right. \\
& \left.+\frac{1}{\omega^{2}} \tau_{p}^{2} \operatorname{det} \tilde{G}_{i j} \tilde{G}^{i j} \mathbf{a}_{j i}\right) \\
& -\lambda^{i}\left(p_{\mu} \partial_{i} X^{\mu}+p_{M} \partial_{i} X^{M}\right),
\end{aligned}
$$

so that we can easily take the limit $\omega \rightarrow \infty$ and we obtain

$$
\begin{aligned}
\mathcal{L}= & p_{\mu} \partial_{0} X^{\mu}+p_{M} \partial_{0} X^{M} \\
& -\lambda^{\tau}\left(p_{M} p^{M}+\tau_{p}^{2} \operatorname{det} \tilde{G}_{i j}\right) \\
& -\lambda^{i}\left(p_{\mu} \partial_{i} X^{\mu}+p_{M} \partial_{i} X^{M}\right) .
\end{aligned}
$$

It is easy to see that this Lagrangian density is invariant under the transformations

$$
\begin{aligned}
\delta X^{\mu} & =\omega_{\nu}^{\mu} X^{v}, \delta X^{M}=\omega_{N}^{M} X^{N}+\lambda_{\nu}^{M} X^{\nu}, \\
\delta p_{\mu} & =-p_{\nu} \omega_{\mu}^{\nu}-p_{M} \lambda_{\mu}^{M}, \quad \delta p_{M}=-p_{N} \omega_{M}^{N},
\end{aligned}
$$

using the fact that in the limit $\omega \rightarrow \infty$ we have the following conditions:

$\omega_{\rho \sigma}+\omega_{\sigma \rho}=0, \omega_{K L}+\omega_{L K}=0$.
As the next step we determine the canonical equations of motion from an extended Hamiltonian,

$$
\begin{aligned}
H= & \int d^{p} \xi\left(\lambda^{\tau}\left(p_{M} p^{M}+\tau_{p}^{2} \operatorname{det} \tilde{G}_{i j}\right)\right. \\
& \left.+\lambda^{i}\left(p_{\mu} \partial_{i} X^{\mu}+p_{M} \partial_{i} X^{M}\right)\right)
\end{aligned}
$$

so that we have the following collection of the canonical equations of motion:

$$
\begin{aligned}
\partial_{0} X^{M}= & \left\{X^{M}, H\right\}=2 \lambda^{\tau} p^{M}+\lambda^{i} \partial_{i} X^{M}, \\
\partial_{0} p_{M}= & \left\{p_{M}, H\right\}=\partial_{i}\left(\lambda^{i} p_{M}\right), \\
\partial_{0} X^{\mu}= & \left\{X^{\mu}, H\right\}=\lambda^{i} \partial_{i} X^{\mu}, \\
\partial_{0} p_{\mu}= & \left\{p_{\mu}, H\right\}=\partial_{i}\left[2 \lambda^{\tau} \tau_{p}^{2} \partial_{j} X_{\mu} \tilde{G}^{j i} \operatorname{det} \tilde{G}_{i j}\right] \\
& +\partial_{i}\left[\lambda^{i} p_{\mu}\right], \\
& p_{M} p^{M}+\tau_{p}^{2} \operatorname{det} \tilde{G}_{i j}=0, \\
& p_{\mu} \partial_{i} X^{\mu}+p_{M} \partial_{i} X^{M}=0 .
\end{aligned}
$$

Let us try to solve these equations of motion at the spatial gauge when $X^{i}=\xi^{i}$. Then the equation of motion for $X^{i}$ implies $\lambda^{i}=0$ and the equation of motion for $p_{M}$ implies that $p_{M}$ can depend on $\xi^{i}$ only. Further we see from the Hamiltonian constraint that the only possibility is to demand that $t$ and $X^{M}, p_{M}$ depend on $\xi^{i}$. Without loss of generality we presume that it depends on $\xi^{1}$ only and hence the matrix $\tilde{G}_{i j}$ is diagonal, in the form

$\tilde{G}_{i j}=\operatorname{diag}\left(1-t^{\prime 2}, 1, \ldots, 1\right)$.

Then the equation of motions for $p_{i}$ are automatically satisfied for $i \neq 1$ and imply $p_{i}=0$, which is also in agreement with the spatial diffeomorphism constraints that imply $p_{i}=-p_{M} \partial_{i} X^{M}$. For $i=1$ the equation of motion for $p_{1}$ has the form

$\partial_{0} p_{1}=\partial_{1}\left[2 \lambda^{\tau} \tau_{p}^{2}\right]$,

which determines the value of the Lagrange multiplier $\lambda^{\tau}$ since $p_{1}=-p_{M} \partial_{1} X^{M}$ and since $p_{M}$ depends on $x$ only:

$2 \frac{\partial_{1}^{2} t}{\partial_{1} t}=\frac{\partial_{1} \lambda^{\tau}}{\lambda^{\tau}}$,

which has the solution

$\lambda^{\tau}=C \partial_{1} t^{2}$,

where $C$ is a constant. Using this result we finally find that $X^{M}=2 C \partial_{1} t^{2} p_{M} \xi^{0}+k^{M}$ where $k^{M}$ can depend on $\xi^{1}$ at least in principle. We see that the non-relativistic p-brane moves freely in the transverse space where, however, the coordinates depend on $\xi^{1}$ through the function $\partial_{1} t$. The simplest possibility is to choose $t=k \xi^{1}$ where $k$ has to obey the 
condition $k>1$. Then we can choose $\lambda^{\tau}=1$ for $C=\frac{1}{k^{2}}$ and $X^{M}$ has the following time dependence:

$X^{M}=2 p^{M} \xi^{0}, \quad p_{M} p^{M}=\tau_{p}^{2}\left(k^{2}-1\right)$.

Let us now determine the Lagrangian for this nonrelativistic p-brane. In fact, using the equations of motion for $X^{M}$ and $X^{\mu}$ we easily find the corresponding Lagrangian density,

$$
\begin{aligned}
\mathcal{L}= & \frac{1}{4 \lambda^{\tau}}\left(\partial_{0} X^{M}-\lambda^{i} \partial_{i} X^{M}\right)\left(\partial_{0} X_{M}-\lambda^{j} \partial_{j} X_{M}\right) \\
& -\lambda^{\tau} \tau_{p}^{2} \operatorname{det} \tilde{G}_{i j} .
\end{aligned}
$$

As the next step we eliminate the Lagrange multipliers using the corresponding equations of motion,

$$
\begin{aligned}
\frac{1}{4\left(\lambda^{\tau}\right)^{2}}\left(\partial_{0} X^{M}-\lambda^{i} \partial_{i} X^{M}\right)^{2}+\tau_{p}^{2} \operatorname{det} \tilde{G}_{i j} & =0, \\
\partial_{i} X^{M}\left(\partial_{0} X_{M}-\lambda^{j} \partial_{j} X_{M}\right) & =0 .
\end{aligned}
$$

To proceed let us analyze the matrix $F_{i j}=\partial_{i} X^{M} \partial_{j} X_{M}$. This is a $p \times p$ matrix which is given as a product of $p \times(d-q)$ matrices $\partial_{i} X^{M}$ and a $(d-q) \times(d-q)$ matrix $\delta_{M N}$ and hence has the rank $\min (p,(d-q))$. This matrix will be nonsingular if $p<d-q$, which leads to the condition (using the fact that $q=p-1$ )

$p<\frac{d+1}{2}$.

Let us presume this case; hence we can solve the last equation in (37) for $\lambda^{i}$, thus

$\lambda^{i}=F_{0 j} F^{j i}, \quad F_{\alpha \beta} \equiv \partial_{\alpha} X^{M} \partial_{\beta} X_{M}$,

so that the first equation in (37) gives

$$
\begin{aligned}
\left(\lambda^{\tau}\right)^{2} & =-\frac{1}{4 \tau_{p}^{2} \operatorname{det} \tilde{G}_{i j}}\left(F_{00}-F_{0 k} F^{k j} F_{j 0}\right) \\
& =-\frac{1}{4 \tau_{p}^{2} \operatorname{det} \tilde{G}_{i j} \operatorname{det} F_{i j}} \operatorname{det} F_{\mu \nu},
\end{aligned}
$$

and hence the Lagrangian density has the final form

$\mathcal{L}=\tau_{p} \sqrt{-\operatorname{det} \tilde{G}_{i j} \frac{\operatorname{det} F_{\alpha \beta}}{\operatorname{det} F_{i j}}}$.

Of course, the last condition holds on condition that the matrix $F_{\alpha \beta}=\partial_{\alpha} X^{M} \partial_{\beta} X_{M}$ is non-singular. On the other hand we see that this is a $(p+1) \times(p+1)$ matrix with the rank given as $\min (p+1, d-(p-1))$, which implies

$p<\frac{d}{2}$,

which is a stronger condition than the condition (38) but certainly can be obeyed. Finally, note that (41) is invariant under the scaling transformations

$X^{\prime \mu}=\lambda^{-\frac{1}{p}} X^{\mu}, \quad X^{\prime M}=\lambda X^{M}$.
It is interesting that the Lagrangian density derived from the Hamiltonian is not unique. For example, let us consider an equation of motion for $X^{\mu}$,

$\partial_{0} X^{\mu}=\lambda^{i} \partial_{i} X^{\mu}$.

Multiplying this equation with $\partial_{j} X_{\mu}$ we obtain

$\partial_{0} X^{\mu} \partial_{j} X_{\mu}=\lambda^{i} \tilde{G}_{i j}$,

which can be solved for $\lambda^{i}$ using the fact that $\tilde{G}_{i j}$ is nonsingular,

$\lambda^{i}=\tilde{G}^{i j} \tilde{G}_{j 0}, \quad \tilde{G}_{\alpha \beta}=\partial_{\alpha} X^{\mu} \partial_{\beta} X_{v}$.

Then the equation of motion for $\lambda^{\tau}$ has the form

$$
\begin{aligned}
\left(\lambda^{\tau}\right)^{2}= & -\frac{1}{4 \tau_{p}^{2} \operatorname{det} \tilde{G}_{i j}}\left(\mathbf{a}_{00}-2 \tilde{G}_{0 j} \tilde{G}^{j i} \mathbf{a}_{i 0}\right. \\
& \left.+\tilde{G}_{0 i} \tilde{G}^{i k} \mathbf{a}_{k l} \tilde{G}^{l j} \tilde{G}_{j 0}\right),
\end{aligned}
$$

and hence we obtain the following Lagrangian density:

$$
\mathcal{L}=-\tau_{p} \sqrt{\left(\mathbf{a}_{00}-2 \tilde{G}_{0 j} \tilde{G}^{j i} \mathbf{a}_{j 0}+\tilde{G}_{0 i} \tilde{G}^{i k} \mathbf{a}_{k l} \tilde{G}^{l j} \tilde{G}_{j 0}\right) \operatorname{det} \tilde{G}_{i j}} .
$$

We argued that the form of non-relativistic Lagrangian density depends on the value of the coefficient $k_{q}$. Let us consider the second possibility, that $k_{q}$ is equal to $k_{q}=p-1$, so that the Lagrangian density is equal to

$$
\begin{aligned}
\mathcal{L}= & p_{\mu} \partial_{0} X^{\mu}+p_{M} \partial_{0} X^{M} \\
& -\lambda^{\tau}\left(\frac{1}{\omega^{2}} p_{\mu} \eta^{\mu \nu} p_{v}\right. \\
& \left.+p_{M} p^{M}+\omega^{2} \tau_{p}^{2} \operatorname{det} \tilde{G}_{i j}+\tau_{p}^{2} \operatorname{det} \tilde{G}_{i j} \tilde{G}^{i j} \mathbf{a}_{j i}\right) \\
& -\lambda^{i}\left(p_{\mu} \partial_{i} X^{\mu}+p_{M} \partial_{i} X^{M}\right),
\end{aligned}
$$

so that we can easily take the limit $\omega \rightarrow \infty$ and we obtain

$$
\begin{aligned}
\mathcal{L}= & \omega^{2} \mathcal{L}_{2}+\mathcal{L}_{0}, \quad \mathcal{L}_{2}=-\lambda^{\tau} \tau_{p}^{2} \operatorname{det} \tilde{G}_{i j}, \\
\mathcal{L}_{0}= & p_{\mu} \partial_{0} X^{\mu}+p_{M} \partial_{0} X^{M} \\
& -\lambda^{\tau}\left(p_{M} p^{M}+\tau_{p}^{2} \operatorname{det} \tilde{G}_{i j} \tilde{G}^{i j} \mathbf{a}_{i j}\right) \\
& -\lambda^{i}\left(p_{\mu} \partial_{i} X^{\mu}+p_{M} \partial_{i} X^{M}\right) .
\end{aligned}
$$

Now we would like to analyze transformation rules for different terms in the Lagrangian. Clearly we have $\delta_{0} \mathcal{L}_{2}=0$, while

$$
\begin{aligned}
\delta_{0} \mathcal{L}_{0}= & -\tau_{p}^{2} \operatorname{det} \tilde{G}_{i j} \delta \mathbf{a}_{i j} \\
= & -\tau_{p}^{2} \operatorname{det} \tilde{G}_{i j} \tilde{G}^{i j}\left(\lambda_{\nu}^{M} \partial_{i} X^{\nu} \delta_{M N} \partial_{j} X^{N}\right. \\
& \left.+\partial_{i} X^{M} \delta_{M N} \lambda_{\nu}^{N} \partial_{j} X^{\nu}\right) .
\end{aligned}
$$

On the other hand it is easy to see that

$$
\begin{aligned}
\delta_{-2} \mathcal{L}_{2}= & -\tau_{p}^{2} \operatorname{det} \tilde{G}_{k l} \delta_{(-2)} \tilde{G}_{i j} \tilde{G}^{j i} \\
= & -\tau_{p}^{2} \operatorname{det} \tilde{G}_{k l} \tilde{G}^{i j}\left(\partial_{i}\left(\lambda^{\mu}{ }_{M} X^{M}\right) \eta_{\mu \nu} \partial_{j} X^{\nu}\right. \\
& \left.+\partial_{i} X^{\mu} \eta_{\mu \nu} \partial_{j}\left(\lambda^{\nu}{ }_{M} X^{M}\right)\right),
\end{aligned}
$$


using the fact that $\delta_{-2} X^{\mu}=\Lambda^{\mu}{ }_{M} X^{M}$. Now it is easy to see that $\delta_{-2} \mathcal{L}_{2}+\delta_{0} \mathcal{L}_{0}=0$ thanks to the conditions

$\lambda^{\mu}{ }_{M} \eta_{\mu \rho}+\delta_{M K} \lambda_{\rho}^{K}=0, \quad \eta_{\rho \nu} \lambda^{\nu}{ }_{M}+\lambda_{\rho}^{K} \delta_{K M}=0$.

It is important to stress that the variation of the Lagrangian density $\mathcal{L}_{0}$, proportional to $\lambda_{\mu}^{M}$, is compensated by the variation of the Lagrangian density $\mathcal{L}_{2}$, which is in agreement with [1]. On the other hand it is not completely clear how to deal with the presence of the divergent term in the Lagrangian when we analyze the corresponding equations of motion. The well-defined procedure to eliminate this term is to couple the p-brane to the background $p+1$ form. We will show that this can be done in the canonical approach too.

\section{Elimination of divergent term}

We begin this section with the case of the massive relativistic particle action in the form

$S=\int \mathrm{d} \tau\left(p_{t} \dot{t}+p_{M} \dot{X}^{M}-e\left(-\frac{1}{\omega^{2}} p_{t}^{2}+p_{M}^{2}+\tilde{m}^{2}\right)\right)$,

and we see that the limit $\omega \rightarrow \infty$ gives the result

$S=\int \mathrm{d} \tau\left(p_{t} \dot{t}+p_{M} \dot{X}^{M}-e\left(p_{M}^{2}+\tilde{m}^{2}\right)\right)$.

From the Hamiltonian constraint $p_{M}^{2}+m^{2}=0$ we see that the non-relativistic limit can be defined if we scale $\tilde{m}^{2}=$ $\frac{1}{\omega^{2}} m^{2}$. On the other hand the dynamics is still trivial since the Hamiltonian constraint implies $p_{M} p^{M}=0$, which has the solution $p_{M}=0$. In order to resolve this problem let us consider the possibility that we couple the particle to the background electromagnetic field so that the action has the form

$S=-\tilde{m} \int \mathrm{d} \tau \sqrt{-g_{A B} \dot{X}^{A} \dot{X}^{B}}+M \int \mathrm{d} \tau A_{A} \dot{X}^{A}$,

thus the conjugate momentum is

$p_{A}=\frac{\tilde{m} g_{A B} \dot{X}^{B}}{\sqrt{-g_{A B} \dot{X}^{A} \dot{X}^{B}}}+M A_{A}$,

which implies the following constraint:

$\mathcal{H}_{\tau}=\left(p_{A}-M A_{A}\right) g^{A B}\left(p_{B}-M A_{B}\right)+\tilde{m}^{2} \approx 0$.

We define a non-relativistic limit when $g_{A B}=\operatorname{diag}$ $\left(-\omega^{2}, 1, \ldots, 1\right), g^{A B}=\operatorname{diag}\left(-\frac{1}{\omega^{2}}, 1, \ldots, 1\right)$ and also $A_{0}=$ $\omega^{2}$. Then the Hamiltonian constraint has the form

$\mathcal{H}_{\tau}=p_{M} p^{M}-\frac{1}{\omega^{2}} p_{t}^{2}+2 M p_{t}-M^{2} \omega^{2}+\tilde{m}^{2} \approx 0$.

Now we see that we derive a well-defined limit when we scale $\tilde{m}^{2}$ as $\tilde{m}^{2}=M^{2} \omega^{2}$ so that the Hamiltonian for the non-relativistic particle has the form
$\mathcal{H}_{\tau}=p_{M} p^{M}+2 M p_{t} \approx 0$

This is clearly a non-relativistic Hamiltonian constraint and we see that it was crucial that the particle is coupled to the gauge field. It is now easy to determine the corresponding Lagrangian density using the equation of motion for $X^{M}$ and for $t$,

$\dot{X}^{M}=\left\{X^{M}, H\right\}=2 e p_{M}, \quad \dot{t}=\{t, H\}=2 M e$,

and hence

$$
\begin{aligned}
L & =p_{M} \dot{X}^{M}+p_{t} \dot{t}-H=e p_{M} p^{M} \\
& =\frac{1}{4 e} \dot{X}^{M} \dot{X}_{M}=\frac{M}{2 \dot{t}} \dot{X}^{M} \dot{X}_{M},
\end{aligned}
$$

where in the last step we used the equation of motion for $t$. Note that this Lagrangian has the same form as the Lagrangian for a non-relativistic particle as derived in [28].

As the next step we consider a fundamental string coupled to a background NSNS two form where the action has the form

$$
\begin{aligned}
S= & -\tilde{\tau}_{F} \int \mathrm{d} \tau \mathrm{d} \sigma \sqrt{-\operatorname{det} g_{\alpha \beta}} \\
& +\tilde{\tau}_{F} \int \mathrm{d} \tau \mathrm{d} \sigma B_{A B} \partial_{\tau} X^{A} \partial_{\sigma} X^{B},
\end{aligned}
$$

so that we have the following conjugate momenta:

$$
\begin{aligned}
p_{A}= & -\tilde{\tau}_{F} G_{A B} \partial_{\alpha} X^{B} g^{\beta \tau} \sqrt{-\operatorname{det} g_{\alpha \beta}} \\
& +\tilde{\tau}_{F} B_{A B} \partial_{\sigma} X^{B}
\end{aligned}
$$

which implies the following Hamiltonian constraint:

$$
\begin{aligned}
\mathcal{H}_{\tau}= & \left(p_{A}-\tilde{\tau}_{F} B_{A C} \partial_{\sigma} X^{C}\right) g^{A B}\left(p_{B}-\tilde{\tau}_{F} B_{B D} \partial_{\sigma} X^{D}\right) \\
& +\tilde{\tau}_{F}^{2} G_{A B} \partial_{\sigma} X^{A} \partial_{\sigma} X^{B} \approx 0 .
\end{aligned}
$$

In order to define a stringy non-relativistic limit we choose the components of the metric $G_{\mu \nu}=\omega^{2} \eta_{\mu \nu}, G^{\mu \nu}=$ $\frac{1}{\omega^{2}} \eta^{\mu \nu}, \mu, \nu=0,1$, so that we obtain a Hamiltonian constraint in the form

$$
\begin{aligned}
\mathcal{H}_{\tau}= & \frac{1}{\omega^{2}} p_{\mu} \eta^{\mu \nu} p_{\nu}-2 p_{\mu} \tilde{\tau}_{F} \frac{1}{\omega^{2}} \eta^{\mu v} B_{\nu \sigma} \partial_{\sigma} X^{\sigma} \\
& +p_{M} g^{M N} p_{N}++\frac{1}{\omega^{2}} \tilde{\tau}_{F}^{2} B_{\mu \rho} \partial_{\sigma} X^{\rho} \eta^{\mu v} B_{\nu \sigma} \partial_{\sigma} X^{\sigma} \\
& +\tilde{\tau}_{F}^{2} \omega^{2} \eta_{\mu \nu} \partial_{\sigma} X^{\mu} \partial_{\sigma} X^{\nu}+\tilde{\tau}_{F}^{2} \partial_{\sigma} X^{M} \partial_{\sigma} X_{M} \approx 0 .
\end{aligned}
$$

We see that the divergent term can be eliminated by a suitable choice of the background NSNS two form when we take $B_{\mu \nu}=\omega^{2} \epsilon_{\mu \nu}, \epsilon_{01}=-1$. Further, the string tension is not rescaled $\tilde{\tau}_{F}=\tau_{F}$ and hence the Hamiltonian constraint has the form

$$
\begin{aligned}
\mathcal{H}_{\tau}= & -2 \tau_{F} p_{\mu} \eta^{\mu v} \epsilon_{\nu \sigma} \partial_{\sigma} X^{\sigma}+p_{M} p^{M} \\
& +\tau_{F}^{2} \partial_{\sigma} X^{M} \partial_{\sigma} X_{M} \approx 0 .
\end{aligned}
$$

This is the same form of the Hamiltonian constraint as was derived in [27]. 
The generalization of this procedure to the case of $p$-brane is straightforward. We presume that this $p$-brane couples to a $C^{p+1}$ form so that the action has the form

$$
S=-\tilde{\tau}_{p} \int d^{p+1} \xi \sqrt{-\operatorname{det} \mathbf{A}_{\alpha \beta}}+\tilde{\tau}_{p} \int C^{(p+1)},
$$

where

$$
\begin{aligned}
C^{(p+1)}= & C_{A_{1} \ldots A_{p+1}} d X^{A_{1}} \wedge \ldots d X^{A_{p+1}} \\
= & \frac{1}{(p+1) !} \epsilon^{\alpha_{1} \ldots \alpha_{p+1}} C_{A_{1} \ldots A_{p+1}} \\
& \partial_{\alpha_{1}} X^{A_{1}} \ldots \partial_{\alpha_{p+1}} X^{A_{p+1}} .
\end{aligned}
$$

Thus we have the following conjugate momenta:

$$
\begin{aligned}
p_{A}= & -\tilde{\tau}_{p} G_{A B} \partial_{\beta} X^{B}\left(\mathbf{A}^{-1}\right)^{\beta 0} \sqrt{-\operatorname{det} \mathbf{A}} \\
& +\frac{\tilde{\tau}_{p}}{p !} C_{A A_{2} \ldots A_{p+1}} \epsilon^{i_{2} \ldots i_{p+1}} \partial_{i_{2}} X^{A_{2}} \ldots \partial_{i_{p+1}} X^{A_{p+1}} .
\end{aligned}
$$

Then it is easy to see that the Hamiltonian constraint has the form

$$
\begin{aligned}
\mathcal{H}_{\tau}= & \left(p_{A}-\frac{\tilde{\tau}_{p}}{p !} C_{A A_{2} \ldots A_{p+1}} \epsilon^{i_{2} \ldots i_{p+1}} \partial_{i_{2}} X^{A_{2}}\right. \\
& \left.\ldots \partial_{i_{p+1}} X^{A_{p+1}}\right) G^{A B} \\
& \times\left(p_{B}-\frac{\tilde{\tau}_{p}}{p !} C_{B B_{2} \ldots B_{p+1}} \epsilon^{j_{2} \ldots j_{p+1}} \partial_{j_{2}} X^{B_{2}} \ldots \partial_{j_{p+1}} X^{B_{p+1}}\right) \\
& +\tilde{\tau}_{p}^{2} \operatorname{det} \mathbf{A}_{i j} \approx 0 .
\end{aligned}
$$

Now we presume that the metric has the form $G_{\mu \nu}=$ $\omega^{2} \eta_{\mu \nu}, G_{M N}=\delta_{M N}, G^{\mu \nu}=\frac{1}{\omega^{2}} \eta^{\mu \nu}, \mu, \nu=0, \ldots, p$ so that we have

$$
\operatorname{det} \mathbf{A}_{i j}=\omega^{2 p} \operatorname{det} \tilde{G}_{i j}+\omega^{2 p-2} \operatorname{det} \tilde{G}_{i j} \tilde{G}^{k l} \mathbf{a}_{k l},
$$

and hence we obtain a finite result if $\tilde{\tau}_{p}^{2} \omega^{2(p-1)}=\tau_{p}^{2}$ and if we choose the components of the $p+1$ form along the $0, \ldots, p$ directions in the form

$C_{\mu_{0} \ldots \mu_{p+1}}=\omega^{p+1} \epsilon_{\mu_{0} \mu_{1} \ldots \mu_{p+1}}$.

To proceed we use the fact that

$$
\begin{aligned}
& \frac{\tilde{\tau}_{p}^{2} \omega^{2 p}}{(p !)^{2}} C_{\mu \mu_{2} \ldots \mu_{p+1}} \epsilon^{i_{2} \ldots i_{p+1}} \partial_{i_{2}} X^{\mu_{2}} \\
& \ldots \partial_{i_{p+1}} X^{\mu_{p+1}} \eta^{\mu \nu} C_{\nu v_{2} \ldots v_{p+1}} \epsilon^{j_{2} \ldots j_{p+1}} \partial_{j_{2}} X^{\nu_{2}} \\
& \ldots \partial_{j_{p+1}} X^{v_{p+1}} \\
= & -\frac{\tilde{\tau}_{p}^{2} \omega^{2 p}}{p !} \epsilon^{i_{1} \ldots i_{p}} \epsilon^{j_{1} \ldots j_{p}} \partial_{i_{1}} X^{\mu_{1}} \partial_{j_{1}} X_{\mu_{1}} \ldots \tilde{G}_{i_{1} j_{1}} \ldots \tilde{G}_{i_{p} j_{p}} \\
= & -\tilde{\tau}_{p}^{2} \omega^{2 p} \operatorname{det} \tilde{G}_{i j},
\end{aligned}
$$

and we see that these two divergent contributions cancel. In other words we have the following final form of the Hamiltonian constraint:

$$
\begin{aligned}
\mathcal{H}_{\tau}= & -2 \frac{\tau_{p}}{p !} p^{\mu} \epsilon_{\mu \mu_{2} \ldots \mu_{p+1}} \epsilon^{i_{2} \ldots i_{p+1}} \partial_{i_{2}} X^{\mu_{2}} \ldots \partial_{i_{p+1}} X^{\mu_{p+1}} \\
& +p_{M} p^{M}+\tau_{p}^{2} \operatorname{det} \tilde{G}_{i j} \tilde{G}^{k l} \mathbf{a}_{k l} \approx 0
\end{aligned}
$$

and we see that this Hamiltonian constraint is linear in the non-relativistic momenta. Note that the Hamiltonian constraint is invariant under the transformations

$\delta X^{\mu}=\omega^{\mu}{ }_{\nu}{ }^{\nu}, \quad \delta X^{M}=\omega_{N}^{M} X^{N}$,

where we observe the important fact that the mixed term $\lambda_{\mu}^{M} X^{\mu}$ is absent in the variation of $X^{M}$. This is a consequence of the fact that the presence of the background $p+1$ form breaks the original Lorentz symmetry to the transformations (76), which leaves the background $p+1$ form (73) invariant.

It is instructive to compare the Hamiltonian constraint (75) with the one that was derived in [5] where a Hamiltonian analysis of a non-BPS Dp-brane was performed. The Hamiltonian constraint derived in [5] can be easily truncated to the case of a p-brane and we obtain

$$
\begin{aligned}
\mathcal{H}_{\tau}^{s q . r .}= & p_{M} p^{M}+\tau_{p}^{2} \operatorname{det} \tilde{G}_{i j} \tilde{G}^{k l} \mathbf{a}_{k l} \\
& -\tau_{p} \sqrt{-p_{\mu}\left(\eta^{\mu \nu}-\partial_{i} X^{\mu} \tilde{G}^{i j} \partial_{j} X^{\nu}\right) p_{\nu} .}
\end{aligned}
$$

Since we are interested in the physical content of the theory it is natural to consider the gauge fixed theory and hence we impose the spatial static gauge

$X^{i}=\xi^{i}$,

so that $\tilde{G}_{i j}=\delta_{i j}$ and hence (75) is equal to

$\mathcal{H}_{\tau}=-2 \tau_{p} p_{0}+p_{M} p^{M}+\tau_{p}^{2} \delta^{i j} \mathbf{a}_{i j} \approx 0$.

This agrees with $\mathcal{H}_{\tau}^{\text {sq.r }}$ evaluated at the spatial static gauge too and hence we see that these two Hamiltonian constraints are equivalent.

\section{Particle limit of p-brane action}

Finally, we consider the case of a particle-like non-relativistic limit of the p-brane action where only the time direction is large,

$\tilde{x}^{0}=\omega t, \quad \tilde{x}^{M}=X^{M}, \quad M=1, \ldots, d$,

$\tilde{\tau}_{p}=\frac{1}{\omega} \tau_{p}$.

In this case we have the following primary constraints:

$$
\begin{aligned}
\mathcal{H}_{i} & =p_{t} \partial_{i} t+p_{M} \partial_{i} X^{M}=0, \\
\mathcal{H}_{\tau} & =-\frac{1}{\omega^{2}} p_{t}^{2}+p_{M} p^{M}+\frac{\tau_{p}^{2}}{\omega^{2}} \operatorname{det} \mathbf{A}_{i j} \approx 0,
\end{aligned}
$$


where $\mathbf{A}_{i j}=-\omega^{2} \partial_{i} t \partial_{j} t+\mathbf{a}_{i j}$. Now we can write

$$
\begin{aligned}
\operatorname{det} \mathbf{A}_{i j}= & \frac{1}{p !} \epsilon^{i_{1} \ldots i_{p}} \epsilon^{j_{1} \ldots j_{p}}\left(-\omega^{2} \partial_{i_{1}} t \partial_{j_{1}} t+\mathbf{a}_{i_{1} j_{1}}\right) \\
& \times \ldots\left(-\omega^{2} \partial_{i_{p}} t \partial_{j_{p}} t+\mathbf{a}_{i_{p} j_{p}}\right) \\
= & -\omega^{2} \frac{1}{(p-1) !} \epsilon^{i_{1} \ldots i_{p}} \epsilon^{j_{1} \ldots j_{p}} \partial_{i_{1}} t \partial_{j_{1}} t \mathbf{a}_{i_{2} j_{2}} \\
& \ldots \mathbf{a}_{i_{p} j_{p}}+\operatorname{det} \mathbf{a},
\end{aligned}
$$

where all terms of higher order in $\omega^{2}$ vanish due to the antisymmetry of $\epsilon^{i_{1} \ldots i_{p}}$. Then it is easy to see that the Hamiltonian constraint has the form

$$
\begin{aligned}
\mathcal{H}_{\tau}= & -\frac{1}{\omega^{2}} p_{t}^{2}+p_{M} p^{M} \\
& -\frac{\tau_{p}^{2}}{(p-1) !} \epsilon^{i_{1} \ldots i_{p}} \epsilon^{j_{1} \ldots j_{p}} \partial_{i_{1}} t \partial_{j_{1}} t \mathbf{a}_{i_{2} j_{2}} \ldots \mathbf{a}_{i_{p} j_{p}} \\
& +\frac{\tau_{p}^{2}}{\omega^{2}} \operatorname{det} \mathbf{a} \approx 0,
\end{aligned}
$$

and we see that it is well defined for $\omega \rightarrow \infty$ when we obtain

$$
\begin{aligned}
\mathcal{H}_{\tau}= & p_{M} p^{M}-\frac{\tau_{p}^{2}}{(p-1) !} \epsilon^{i_{1} \ldots i_{p}} \epsilon^{j_{1} \ldots j_{p}} \partial_{i_{1}} t \partial_{j_{1}} t \mathbf{a}_{i_{2} j_{2}} \\
& \ldots \mathbf{a}_{i_{p} j_{p}} \approx 0 .
\end{aligned}
$$

It is easy to see that the Hamiltonian constraint is invariant under non-relativistic transformations,

$\delta p_{M}=-\omega_{M}^{N} p_{N}, \quad \delta X^{M}=\omega_{N}^{M} X^{N}+\lambda^{M} t$

using the fact that $\delta a_{i j}=\lambda^{M}\left(\partial_{i} t \partial_{j} X_{M}+\partial_{i} X_{M} \partial_{j} t\right)$ and then using the antisymmetry of $\epsilon^{i_{1} \ldots i_{p}}$.

It is also instructive to determine the corresponding Lagrangian. Note that the total Hamiltonian has the form

$H=\int d^{p} \xi\left(\lambda^{\tau} \mathcal{H}_{\tau}+\lambda^{i} \mathcal{H}_{i}\right)$

so that we have the following equations of motion:

$$
\begin{aligned}
\partial_{0} X^{M} & =\left\{X^{M}, H\right\}=2 \lambda^{\tau} p^{M}+\lambda^{i} \partial_{i} X^{N}, \\
\partial_{0} t & =\{t, H\}=\lambda^{i} \partial_{i} t
\end{aligned}
$$

and hence the Lagrangian density has the form

$$
\begin{aligned}
\mathcal{L}= & p_{M} \partial_{0} X^{M}+p_{t} \partial_{0} t-\lambda^{\tau} \mathcal{H}_{\tau}-\lambda^{i} \mathcal{H}_{i} \\
= & \frac{1}{4 \lambda^{\tau}}\left(\mathbf{a}_{00}-2 \lambda^{i} \mathbf{a}_{i 0}+\lambda^{i} \lambda^{j} \mathbf{a}_{i j}\right) \\
& +\lambda^{\tau} \frac{\tau_{p}^{2}}{(p-1) !} \epsilon^{i_{1} \ldots i_{p}} \epsilon^{j_{1} \ldots j_{p}} \partial_{i_{1}} t \partial_{j_{1}} t \mathbf{a}_{i_{2} j_{2}} \ldots \mathbf{a}_{i_{p} j_{p}},
\end{aligned}
$$

where again $\mathbf{a}_{\alpha \beta}=\partial_{\alpha} X^{M} \partial_{\beta} X_{M}$. To proceed we solve the equations of motion for $\lambda^{i}$ and $\lambda^{\tau}$,

$$
\begin{aligned}
& \mathbf{a}_{i 0}-\mathbf{a}_{i j} \lambda^{j}=0, \\
& -\frac{1}{4\left(\lambda^{\tau}\right)^{2}}\left(\mathbf{a}_{00}-2 \lambda^{i} \mathbf{a}_{i 0}+\lambda^{i} \lambda^{j} \mathbf{a}_{i j}\right) \\
& +\frac{\tau_{p}^{2}}{(p-1) !} \epsilon^{i_{1} \ldots i_{p}} \epsilon^{j_{1} \ldots j_{p}} \partial_{i_{1}} t \partial_{j_{1}} t \mathbf{a}_{i_{2} j_{2}} \ldots \mathbf{a}_{i_{p} j_{p}}=0 .
\end{aligned}
$$

If we presume that $\mathbf{a}_{i j}$ has an inverse we can find a solution of the first equation as

$\lambda^{i}=\mathbf{a}^{i j} \mathbf{a}_{j 0}$,

so that the equation of motion for $\lambda^{\tau}$ has the form

$$
\begin{aligned}
& -\frac{1}{4\left(\lambda^{\tau}\right)^{2}} \frac{\operatorname{det} \mathbf{a}_{\alpha \beta}}{\operatorname{det} \mathbf{a}_{i j}} \\
& +\frac{\tau_{p}^{2}}{(p-1) !} \epsilon^{i_{1} \ldots i_{p}} \epsilon^{j_{1} \ldots j_{p}} \partial_{i_{1}} t \partial_{j_{1}} t \mathbf{a}_{i_{2} j_{2}} \ldots \mathbf{a}_{i_{p} j_{p}}=0 .
\end{aligned}
$$

Hence the Lagrangian density has the form

$\mathcal{L}=\tau_{p} \sqrt{\frac{\operatorname{det} \mathbf{a}_{\alpha \beta}}{\operatorname{det} \mathbf{a}_{i j}(p-1) !} \epsilon^{i_{1} \ldots i_{p}} \epsilon^{j_{1} \ldots j_{p}} \partial_{i_{1}} t \partial_{j_{1}} t \mathbf{a}_{i_{2} j_{2}} \ldots \mathbf{a}_{i_{p} j_{p}}}$.

It is clear that this analysis is valid for $p>1$. The case $p=1$ will be studied separately in the next subsection.

5.1 The case of fundamental string

In the case of a fundamental string the Hamiltonian constraint has the form

$$
\begin{aligned}
\mathcal{H}_{\tau}= & -\frac{1}{\omega^{2}} p_{t}^{2}+p_{M} p^{M} \\
& -\tau_{F}^{2}\left(\partial_{\sigma} t \partial_{\sigma} t-\frac{1}{\omega^{2}} \partial_{\sigma} X^{M} \partial_{\sigma} X_{M}\right) \approx 0,
\end{aligned}
$$

which implies in the limit $\omega \rightarrow \infty$ the following Hamiltonian constraint:

$\mathcal{H}_{\tau}=p_{M} p^{M}-\tau_{F}^{2} \partial_{\sigma} t \partial_{\sigma} t \approx 0$,

which agrees with the Hamiltonian constraints found in [1].

It is interesting to find the corresponding Lagrangian density. To do this we use again the canonical equations of motion,

$$
\begin{aligned}
\partial_{\tau} X^{M} & =\left\{X^{M}, H\right\}=2 \lambda^{\tau} p_{M}+\lambda^{\sigma} \partial_{\sigma} X^{M}, \\
\partial_{\tau} t & =\{t, H\}=\lambda^{\sigma} \partial_{\sigma} t
\end{aligned}
$$

and hence the Lagrangian density has the form

$$
\begin{aligned}
\mathcal{L}= & p_{M} \partial_{\tau} X^{M}+p_{t} \partial_{\tau} t-\lambda^{\tau} \mathcal{H}_{\tau}-\lambda^{\sigma} \mathcal{H}_{\sigma} \\
= & \frac{1}{4 \lambda^{\tau}}\left(\partial_{\tau} X^{M}-\lambda^{\sigma} \partial_{\sigma} X^{M}\right)\left(\partial_{\tau} X_{M}-\lambda^{\sigma} \partial_{\sigma} X_{M}\right) \\
& +\lambda^{\tau} \tau_{F}^{2} \partial_{\sigma} t \partial_{\sigma} t
\end{aligned}
$$


Solving the equation of motion for $\lambda^{\sigma}$ we obtain

$\lambda^{\sigma}=\frac{\mathbf{a}_{\tau \sigma}}{\mathbf{a}_{\sigma \sigma}}$,

while the equation of motion for $\lambda^{\tau}$ has the form

$$
-\frac{1}{4\left(\lambda^{\tau}\right)^{2}}\left(\mathbf{a}_{\tau \tau}-\frac{\mathbf{a}_{\tau \sigma}^{2}}{\mathbf{a}_{\sigma \sigma}}\right)+\tau_{F}^{2} \partial_{\sigma} t \partial_{\sigma} t=0 .
$$

Inserting this result into the original Lagrangian density we finally obtain

$\mathcal{L}=\tau_{F} \sqrt{\frac{\operatorname{det} \mathbf{a}_{\alpha \beta}}{\mathbf{a}_{\sigma \sigma}} \partial_{\sigma} t \partial_{\sigma} t}$.

It is interesting that this Lagrangian does not have the same form as the Lagrangian found in [1]. Explicitly, the Lagrangian density derived there has the form

$$
\begin{aligned}
\mathcal{L} & =-\tau_{F} \int \mathrm{d} \tau \mathrm{d} \sigma \sqrt{\left(\partial_{\tau} t \partial_{\sigma} X^{M}-\partial_{\sigma} t \partial_{\tau} X^{M}\right)\left(\partial_{\tau} t \partial_{\sigma} X_{M}-\partial_{\sigma} t \partial_{\tau} X_{M}\right)} \\
& =-\tau_{F} \int \mathrm{d} \tau \mathrm{d} \sigma \sqrt{\partial_{\tau} t \partial_{\tau} t \mathbf{a}_{\sigma \sigma}-2 \partial_{\sigma} t \partial_{\tau} t \mathbf{a}_{\tau \sigma}+\partial_{\sigma} t \partial_{\sigma} t \mathbf{a}_{\tau \tau}} \\
& =-\tau_{F} \int \mathrm{d} \tau \mathrm{d} \sigma \sqrt{\mathbf{B}}
\end{aligned}
$$

From (98) we obtain the following conjugate momenta:

$$
\begin{aligned}
p_{t} & =-\tau_{F} \frac{\partial_{\tau} t \mathbf{a}_{\sigma \sigma}-\partial_{\sigma} t \mathbf{a}_{\tau \sigma}}{\sqrt{\mathbf{B}}}, \\
p_{M} & =-\tau_{F} \frac{\partial_{\tau} X_{M} \partial_{\sigma} t \partial_{\sigma} t-\partial_{\sigma} t \partial_{\tau} t \partial_{\sigma} X_{M}}{\sqrt{\mathbf{B}}} .
\end{aligned}
$$

We again find that the bare Hamiltonian is zero, while we have the following collection of the primary constraints:

$$
\begin{aligned}
& \mathcal{H}_{\sigma}=p_{M} \partial_{\sigma} X^{M}+p_{t} \partial_{\sigma} t \approx 0, \\
& \mathcal{H}_{\tau}=p_{M} p^{M}-\tau_{F}^{2} \partial_{\sigma} t \partial_{\sigma} t \approx 0 .
\end{aligned}
$$

It is interesting that in the case of a fundamental string we can find the same form of the Lagrangian density as in [1]. To see this note that the equation of motion for $t$ implies

$\lambda^{\sigma}=\frac{\partial_{\tau} t}{\partial_{\sigma} t}$,

so that the Lagrangian has the form

$$
\begin{aligned}
\mathcal{L}= & \frac{1}{4 \lambda^{\tau}}\left(\mathbf{a}_{\tau \tau}-2 \frac{\partial_{\tau} t}{\partial_{\sigma} t} \mathbf{a}_{\tau \sigma}+\frac{\partial_{\tau} t \partial_{\tau} t}{\partial_{\sigma} t \partial_{\sigma} t} \mathbf{a}_{\sigma \sigma}\right) \\
& +\lambda^{\tau} \tau_{F}^{2} \partial_{\sigma} t \partial_{\sigma} t .
\end{aligned}
$$

Then solving the equation of motion for $\lambda^{\tau}$ we find

$\lambda^{\tau}=-\frac{1}{2 \tau_{F}} \sqrt{\frac{\mathbf{a}_{\tau \tau}-2 \frac{\partial_{\tau} t}{\partial_{\sigma} t} \mathbf{a}_{\tau \sigma}+\frac{\partial_{\tau} t \partial_{\tau} t}{\partial_{\sigma} t \partial_{\sigma} t} \mathbf{a}_{\sigma \sigma}}{\partial_{\sigma} t \partial_{\sigma} t} .}$

Inserting this result back into (102) we finally obtain

$\mathcal{L}=-\tau_{F} \sqrt{\mathbf{a}_{\tau \tau} \partial_{\sigma} t \partial_{\sigma} t-2 \partial_{\tau} t \partial_{\sigma} t \mathbf{a}_{\tau \sigma}+\partial_{\tau} t \partial_{\tau} t \mathbf{a}_{\sigma \sigma}}$,

which agrees with (98).

\section{Conclusion}

Let us outline our results. We proposed different nonrelativistic limits for the p-brane when the relativistic action has the canonical form. We discussed two particular cases where the corresponding Hamiltonian constraint takes a very simple form even if this procedure is completely general and serves as an analogue to the procedure suggested in [1]. On the other hand using the canonical form of the action we easily found dynamical degrees of freedom and the corresponding Hamiltonian. Then when we performed an inverse transformation we derived a Lagrangian density that differs from the Lagrangian density that is derived from the relativistic Lagrangian density by the absence of the kinetic term for non-relativistic coordinates, which is best seen by an example of the particle-like limit of a relativistic p-brane. We showed that these two Lagrangian densities agree in the case of a fundamental string as in [1].

This paper could be extended in various ways. For example, it would be nice to analyze a string in a general NewtonCartan background. It would also be nice to extend this analysis to the Green-Schwarz superstring action. We hope to return to these problems in the near future.

Acknowledgements This work was supported by the Grant Agency of the Czech Republic under the Grant P201/12/G028.

Open Access This article is distributed under the terms of the Creative Commons Attribution 4.0 International License (http://creativecomm ons.org/licenses/by/4.0/), which permits unrestricted use, distribution, and reproduction in any medium, provided you give appropriate credit to the original author(s) and the source, provide a link to the Creative Commons license, and indicate if changes were made.

Funded by $\mathrm{SCOAP}^{3}$.

\section{References}

1. C. Batlle, J. Gomis, D. Not, Extended Galilean symmetries of non-relativistic strings. JHEP 1702, 049 (2017). https://doi.org/ 10.1007/JHEP02(2017)049. arXiv:1611.00026 [hep-th]

2. J. Gomis, P.K. Townsend, The Galilean superstring. arXiv: 1612.02759 [hep-th]

3. C. Batlle, J. Gomis, L. Mezincescu, P.K. Townsend, Tachyons in the Galilean limit. JHEP 1704, 120 (2017). https://doi.org/10.1007/ JHEP04(2017)120. arXiv:1702.04792 [hep-th]

4. J. Kluson, Note about integrability of non-relativistic string. arXiv: 1705.10951 [hep-th]

5. J. Kluson, Hamiltonian analysis of non-relativistic non-BPS Dpbrane. arXiv:1704.08003 [hep-th]

6. S.A. Hartnoll, A. Lucas, S. Sachdev, Holographic quantum matter. arXiv: 1612.07324 [hep-th]

7. P. Horava, Quantum gravity at a Lifshitz point. Phys. Rev. D 79, 084008 (2009). https://doi.org/10.1103/PhysRevD.79.084008. arXiv:0901.3775 [hep-th]

8. A. Wang, Hořava gravity at a Lifshitz point: a progress report. Int. J. Mod. Phys. D 26(07), 1730014 (2017). https://doi.org/10.1142/ S0218271817300142. arXiv:1701.06087 [gr-qc] 
9. J. Hartong, Y. Lei, N.A. Obers, Nonrelativistic Chern-Simons theories and three-dimensional Hoava-Lifshitz gravity. Phys. Rev. D 94(6), 065027 (2016). https://doi.org/10.1103/PhysRevD.94. 065027. arXiv:1604.08054 [hep-th]

10. J. Hartong, N.A. Obers, Hoava-Lifshitz gravity from dynamical Newton-Cartan geometry. JHEP 1507, 155 (2015). https://doi.org/ 10.1007/JHEP07(2015)155. arXiv:1504.07461 [hep-th]

11. E. Bergshoeff, J. Gomis, B. Rollier, J. Rosseel, T. ter Veldhuis, Carroll versus Galilei Gravity. arXiv:1701.06156 [hep-th]

12. E.A. Bergshoeff, J. Rosseel, Three-dimensional extended Bargmann supergravity. Phys. Rev. Lett. 116(25), 251601 (2016). https://doi.org/10.1103/PhysRevLett.116.251601. arXiv:1604.08042 [hep-th]

13. J. Hartong, Gauging the Carroll algebra and ultra-relativistic gravity. JHEP 1508, 069 (2015). https://doi.org/10.1007/ JHEP08(2015)069. arXiv:1505.05011 [hep-th]

14. E. Bergshoeff, J. Rosseel, T. Zojer, Newton-Cartan (super)gravity as a non-relativistic limit. Class. Quant. Gravit. 32(20), 205003 (2015). https://doi.org/10.1088/0264-9381/32/20/205003. arXiv:1505.02095 [hep-th]

15. R. Andringa, E. Bergshoeff, S. Panda, M. de Roo, Newtonian gravity and the Bargmann algebra. Class. Quant. Gravit. 28, 105011 (2011). https://doi.org/10.1088/0264-9381/28/10/ 105011. arXiv:1011.1145 [hep-th]

16. E. Bergshoeff, J. Rosseel, T. Zojer, Newton-Cartan supergravity with torsion and Schrdinger supergravity. JHEP 1511, 180 (2015). https://doi.org/10.1007/JHEP11(2015)180. arXiv:1509.04527 [hep-th]

17. E.A. Bergshoeff, J. Hartong, J. Rosseel, Torsional NewtonCartan geometry and the Schrdinger algebra. Class. Quant. Gravit. 32(13), 135017 (2015). https://doi.org/10.1088/0264-9381/32/13/ 135017. arXiv: 1409.5555 [hep-th]

18. J. Gomis, H. Ooguri, Nonrelativistic closed string theory. J. Math. Phys. 42, 3127 (2001). https://doi.org/10.1063/1.1372697. arXiv:hep-th/0009181
19. U.H. Danielsson, A. Guijosa, M. Kruczenski, IIA/B, wound and wrapped. JHEP 0010, 020 (2000). https://doi.org/10.1088/ 1126-6708/2000/10/020. arXiv:hep-th/0009182

20. J. Gomis, J. Gomis, K. Kamimura, Non-relativistic superstrings: a new soluble sector of $\operatorname{AdS}(5)$ x $\mathrm{S} * * 5$. JHEP 0512, 024 (2005). https://doi.org/10.1088/1126-6708/2005/12/ 024. arXiv:hep-th/0507036

21. J. Gomis, K. Kamimura, P.K. Townsend, Non-relativistic superbranes. JHEP 0411, 051 (2004). https://doi.org/10.1088/ 1126-6708/2004/11/051. arXiv:hep-th/0409219

22. J. Kluson, Non-Relativistic Non-BPS Dp-brane. Nucl. Phys. B 765, 185 (2007). https://doi.org/10.1016/j.nuclphysb.2006.12.010. arXiv:hep-th/0610073

23. J. Brugues, T. Curtright, J. Gomis, L. Mezincescu, Non-relativistic strings and branes as non-linear realizations of Galilei groups. Phys. Lett. B 594, 227 (2004). https://doi.org/10.1016/j.physletb.2004. 05.024. arXiv:hep-th/0404175

24. J. Gomis, F. Passerini, T. Ramirez, A. Van Proeyen, Non relativistic Dp branes. JHEP 0510, 007 (2005). https://doi.org/10.1088/ 1126-6708/2005/10/007. arXiv:hep-th/0507135

25. M. Sakaguchi, K. Yoshida, Non-relativistic AdS branes and Newton-Hooke superalgebra. JHEP 0610, 078 (2006). https://doi. org/10.1088/1126-6708/2006/10/078. arXiv:hep-th/0605124

26. M. Sakaguchi, K. Yoshida, Non-relativistic string and D-branes on $\operatorname{AdS}(5) \times \mathrm{S} * * 5$ from semiclassical approximation. JHEP 0705, 051 (2007). https://doi.org/10.1088/1126-6708/2007/05/ 051. arXiv:hep-th/0703061 [HEP-TH]

27. J. Gomis, F. Passerini, Rotating solutions of non-relativistic string theory. Phys. Lett. B 617, 182 (2005). https://doi.org/10.1016/j. physletb.2005.04.061. arXiv:hep-th/0411195

28. R. Andringa, E. Bergshoeff, J. Gomis, M. de Roo, Stringy NewtonCartan gravity. Class. Quant. Gravit. 29, 235020 (2012). https://doi. org/10.1088/0264-9381/29/23/235020. arXiv:1206.5176 [hep-th]

29. T. Harmark, J. Hartong, N.A. Obers, Non-relativistic strings and limits of the AdS/CFT correspondence. arXiv:1705.03535 [hep-th] 